\title{
Editorial: Reporting guidelines for psychopharmacology
}

\author{
Thomas Steckler $^{1}$ - H. Valerie Curran ${ }^{2}$ Harriet de Wit $^{3}$ • Oliver Howes ${ }^{4,5}$. \\ Daniel Hoyer $^{6,7}$ • Irwin Lucki ${ }^{8,9}$ - Klaus A. Miczek ${ }^{10,11,12,13}$ • A. Leslie Morrow ${ }^{14,15}$. \\ Lawrence H. Price ${ }^{16,17}$ • Trevor W. Robbins ${ }^{18,19}$
}

Published online: 26 February 2016

(C) Springer-Verlag Berlin Heidelberg 2016

\section{Introduction}

Robust and generalizable data, and the full and transparent reporting of those data, are key to drawing the right conclusions, deciding on next experimental steps, and achieving scientific progress (Steckler 2015). Notably, many scientific articles, not just those in the field of psychopharmacology, lack sufficient methodological detail (Vasilevsky et al. 2013) and important information on experimental design (Bebarta et al. 2003; Hirst et al. 2014; Iqbal et al. 2016; Kilkenny

Thomas Steckler

TSTECKLE@its.jnj.com

1 Janssen Research \& Development, Turnhoutseweg 30, 2340 Beerse, Belgium

2 Clinical Psychopharmacology Unit, University College London, London WC1E 6BT, UK

3 Department of Psychiatry and Behavioral Neuroscience, University of Chicago, Chicago, IL 60637, USA

4 MRC Clinical Sciences Centre, Imperial College Hammersmith Campus, London SW7 2AZ, UK

5 Institute of Psychiatry, Psychology and Neuroscience, King's College London, 16 De Crespigny Park, London SE5 8AF, UK

6 Department of Pharmacology \& Therapeutics, School of Biomedical Sciences, Faculty of Medicine, Dentistry and Health Sciences, The University of Melbourne, Parkville, VIC 3010, Australia

7 The Florey Institute of Neuroscience and Mental Health, The University of Melbourne, 30 Royal Parade, Parkville, VIC 3052, Australia

8 Department of Psychiatry, University of Pennsylvania, Philadelphia, PA 19104, USA

9 Department of Pharmacology, University of Pennsylvania, Philadelphia, PA 19104, USA et al. 2009; Macleod et al. 2015; McCance 1995; Vesterinen et al. 2011). These details are essential to allow the reader to understand what exactly was done in a study, to judge the quality of the data, and to repeat the study. Accordingly, many scientific journals are emphasizing guidelines for the full and transparent reporting of data (Curtis and Abernethy 2015; Curtis et al. 2015; Kenall et al. 2015; Kilkenny et al. 2011; Nature Publishing Group 2014; Nosek et al. 2015).

To help authors in Psychopharmacology to continue to produce high-quality articles containing all the information

10

Department of Psychology, Tufts University, Bacon Hall, Medford, MA 02155, USA

11 Department of Neuroscience, Tufts University, Bacon Hall, Medford, MA 02155, USA

12 Department of Pharmacology, Tufts University, Bacon Hall, Medford, MA 02155, USA

13 Department of Psychiatry, Tufts University, Bacon Hall, Medford, MA 02155, USA

14 Department of Psychiatry, Bowles Center for Alcohol Studies, University of NC School of Medicine, Chapel Hill, NC 27599-7178, USA

15 Department of Pharmacology, Bowles Center for Alcohol Studies, University of NC School of Medicine, Chapel Hill, NC 27599-7178, USA

16 Department of Psychiatry and Human Behavior, Alpert Medical School, Brown University, Providence, RI, USA

17 Butler Hospital, 345 Blackstone Blvd, Providence, RI 02906, USA

18 Department of Psychology, University of Cambridge, Downing Street, Cambridge CB2 3EB, UK

19 MRC and Wellcome Trust Behavioural and Clinical Neuroscience Institute, University of Cambridge, Cambridge CB2 3EB, UK 
necessary to judge the rigor of a study, to improve reporting practice and to enhance the impact of articles published in the journal, the editors of Psychopharmacology have agreed on the following guidelines, in accordance with Springer's longstanding policy on the full and transparent reporting of data and experimental design:

\begin{abstract}
The Abstract should contain the purpose, methods, results and conclusions. In the results, the effect size should be included if possible.
\end{abstract}

\section{Experimental design and statistics}

The "Methods" section should provide information sufficient to allow replication of the work and full details on the statistical methods used. The figure legends should provide information essential to interpreting the data presented. The following information should be included in the "Methods":

1. The exact sample size ( $n$ ) for each experimental group/ condition (as a number, not a range).

2. An explanation of how the sample size was chosen for each experiment, including power analysis where appropriate.

3. A description of sample collection that enables the reader to understand whether the samples represent technical or biological replicates, and an explanation of inclusion/exclusion criteria if data samples or subjects were excluded from the analysis (outlier criteria).

4. A description of how samples/animals which were allocated to experimental groups and processed, and full details of the randomization procedure used (if relevant).

5. A statement on whether the investigator was blinded to group assignment and outcome assessment, and how this blinding was achieved and evaluated (if relevant).

6. How many times each experiment shown was replicated (if applicable).

7. Primary and secondary endpoints/measures should be specified.

8. Information on the statistical methods and measures used. Authors should indicate whether tests were onesided or two-sided and whether adjustments were made for multiple comparisons. The figure legends should indicate whether medians or means are shown, whether error bars are standard deviations (SD), standard error of mean (SEM) or confidence intervals, and should include the number of data points per group used to generate the figure.

9. A justification for the appropriateness of the statistical test used should be provided, e.g., whether data meet the assumptions of the tests (e.g., normal distribution), whether the variation within each group of data has been estimated, and whether the variance observed is similar between the groups that are being statistically compared.

10. Systematic reviews should follow recognized guidelines on conduct and reporting (e.g., PRISMA: http://www.prismastatement.org/).

If the study involves human participants, authors should refer to the relevant reporting guidelines from the EQUATOR Network: http://www.equator-network.org/.

\section{Availability of data}

The editors support the principle of raw data sharing where possible and feasible, not only for reasons of full traceability and transparency but also to advance open science and to allow better exploitation of the data and more complete extraction of the wealth of information potentially existing in those data sets. At present, different publishers and institutional bodies have different requirements, and many are working on the best solution to data availability, including what exactly to deposit and in what format such deposits should be made (Alsheikh-Ali et al. 2011; Goodwin 2015; Poldrack and Gorgolewski 2014; see also NIH Sharing Policies and Related Guidance on NIH-Funded Research Resources, https://grants.nih.gov/grants/sharing.htm, and the recent announcement of the NIH/Wellcome Trust Open Science Prize, Oct 2015, http://www.nih.gov/news-events/newsreleases/new-prize-competition-seeks-innovative-ideasadvance-open-science). While we realize the practical difficulties that may be associated with this effort, authors are encouraged to deposit key raw and all processed datasets on which the conclusions of the paper rely in publicly available repositories (e.g., using Dryad (http://datadryad. org/), FigShare (http://figshare.com/), the Neuroscience Information Network (http://www.neuinfo.org/about/index. shtm), OpenfMRI (https://openfmri.org/)). Alternatively, the data may be presented in the main paper or supporting files (e.g., as Supplementary Material), in an annotated, machinereadable format whenever possible. Links to deposited datasets or datasets in additional files should be explicitly referenced in a section entitled "Availability of data and materials". At a minimum, authors should be prepared to provide raw data from published studies, if ethically appropriate, to other investigators upon request, or to the editors during the review process, if needed.

The editor may specifically request that data be made publicly available. If data cannot be deposited in response to such a request, reasons should be provided to the editor and in the "Availability of data and materials" section. Under such 
circumstances, the editor will determine whether the manuscript can still be considered for publication.

If computer code was used to generate results that are central to the paper's conclusions, a statement should be included in the "Availability of data and materials" section to indicate whether and how the code can be accessed, including version information as necessary and information on possible restrictions on availability.

Appropriate credit should be given to the originators of the raw data. Third parties using the data for further analysis and publication should cite the source, which could be the publication in Psychopharmacology or elsewhere.

\section{Resources}

A description of all resources used with enough information to be uniquely identified should be included as a "Methods" subsection entitled "Resources".

- Antibodies: source, characteristics, dilutions and how they were validated for the system under study should be reported.

- Cell lines: source, whether identity has been authenticated and whether cell lines were tested for mycoplasma contamination should be reported.

- Animals: source, species, strain, sex, age, husbandry, inbred and strain characteristics of transgenic and mutant animals should be reported.

- Tools (software, databases and services): standard tool names, provider and version number, if available, should be reported.

- Test compounds: source, purity, chemical structure (if not published previously), salt form, formulation, vehicle, relevant pharmacokinetic and pharmacodynamic properties in the relevant species, e.g., plasma and brain concentration, brain penetration, half-life, stability, affinity, selectivity, target engagement (if not published previously) should be reported. For information already reported elsewhere, the relevant references should be provided. If that information is not available, it remains at the discretion of the editor to decide whether this is acceptable. Test compounds should be tested in appropriate concentrations or dose-response.

Work on the actions of biological extracts of unknown chemical composition, i.e., of a mixture of ingredients that is insufficiently defined and/or of unknown concentrations that might affect the results, is normally not considered for publication. Studies using plant materials with unknown or uncontrolled constituents are discouraged. Exceptions will be made if the plant materials are highly standardized and well characterized (e.g., tobacco; cannabis with specified cannabinoid content). If the pharmacological actions of all the relevant components are taken into account, studies with certain biological materials of uncertain composition may be considered for publication in Psychopharmacology.

Authors are also encouraged to provide Research Resource Identifiers (RRIDs) for antibodies, organisms and tools (Resource Identification Portal (https://scicrunch.org/ resources).

While we realize that our new guidelines will cause additional work for authors, we consider these factors to be crucial elements in the reporting of scientific findings in our field and are convinced that the gain of the extra information provided will greatly outweigh this effort and will further increase the impact of the articles published in Psychopharmacology.

Acknowledgments The Reporting Guidelines for Psychopharmacology are modified from guidelines recently published by the BioMed Central Reproducibility Working Group (http://www.genomebiology.com/ authors/instructions/minimum_standards_reporting; see also Kenall et al. 2015).

\section{References}

Alsheikh-Ali AA, Qureshi W, Al-Mallah MH, Ioannidis JPA (2011) Public availability of published research data in high-impact journals. PLoS ONE 6:e24357

Bebarta V, Luyten D, Heard K (2003) Emergency medicine animal research: does use of randomization and blinding affect the results? Acad Emerg Med 10:684-687

Curtis MJ, Abernethy DR (2015) Revision of instructions to authors for pharmacology research and perspectives: enhancing the quality and transparency of published work. Pharmacol Res Perspect 3:e00106

Curtis MJ, Bond RA, Spina D, Ahluwalia A, Alexander SPA, Giembycz MA, Gilchrist A, Hoyer D, Insel PA, Izzo AA, Lawrence AJ, MacEwan DJ, Moon LDF, Wonnacott S, Weston AH, McGrath JC (2015) Experimental design and analysis and their reporting: new guidance for publication in BJP. Br J Pharmacol 172:3461-3471

Goodwin D (2015) Why neuroscience needs hackers. Scientific American, http://www.scientificamerican.com/article/whyneuroscience-needs-hackers

Hirst JA, Howick J, Aronson JK, Roberts N, Koshiaris C, Heneghan C (2014) The need for randomization in animal trials: an overview of systematic reviews. PLoS ONE 9:e98856. doi:10.1371/journal. pone. 0098856

Iqbal SA, Wallach JD, Khoury MJ, Schully SD, Ioannidis JPA (2016) Reproducible research practices and transparency across the biomedical literature. PLoS Biol 14:e1002333. doi:10.1371/journal. pbio. 1002333

Kenall A, Edmunds S, Goodman L, Bal L, Flintoft L, Shanahan DR, Shipley T (2015) Better reporting for better research: a checklist for reproducibility. Genome Biol 16:141. doi:10.1186/s13059-015$0710-5$

Kilkenny C, Parsons N, Kadyszewski E, Festing MFW, Cuthill IC, Fry D, Hutton J, Altman DG (2009) Survey of the quality of experimental design, statistical analysis and reporting of research using animals. PLoS ONE 4:e7824. doi:10.1371/journal.pone.0007824 
Kilkenny C, Browne W, Cuthill IC, Emerson M, Altman DG (2011) Animal research: reporting in vivo experiments - the ARRIVE Guidelines. J Cereb Blood Flow Metab 31:991-993

Macleod MR, McLean AL, Kyriakopoulou A, Serghiou S, de Wilde A, Sherratt N, Hirst T, Hemblade R, Bahor Z, Nunes-Fonseca C, Potluru A, Thomson A, Baginskitae J, Egan K, Vesterinen H, Currie GL, Churilov L, Howells DW, Sena ES (2015) Risk of bias in reports of in vivo research: a focus for improvement. PLoS Biol 13:e1002273. doi:10.1371/journal.pbio.1002273DOI:10.1371/ journal.pbio. 1002273

McCance I (1995) Assessment of statistical procedures used in papers in the Australian Veterinary Journal. Aust Vet J 72:322-330

Nature Publishing Group (2014) Reporting checklist for life sciences articles

Nosek SA, Alter G, Banks GC, Borsboom D, Bowman SD, Breckler SJ, Buck S, Chambers CD, Chin G, Christensen G, Contestabile M, Dafoe A, Eich E, Freese J, Glennerster R, Goroff D, Green DP, Hesse B, Humphreys M, Ishiyama J, Karlan D, Kraut A, Lupia A, Mabry P, Madon TA, Malhotra N, Mayo-Wilson E, McNutt M,
Miguel E, Paluck EL, Simonsohn U, Soderberg C, Spellman BA, Turitto J, VandenBos G, Vazire S, Wagenmakers EJ, Wilson R, Yarkoni T (2015) Promoting an open research culture: author guidelines for journals could help to promote transparency, openness, and reproducibility. Science 348:1422-1425

Poldrack RA, Gorgolewski KJ (2014) Making big data open: data sharing in neuroimaging. Nat Neurosci 17:1510-1517

Steckler T (2015) Preclinical data reproducibility for R\&D-the challenge for neuroscience. Psychopharmacology 232:317320

Vasilevsky NA, Brush MH, Paddock H, Ponting L, Tripathy SJ, LaRocca GL, Haendel MA (2013) On the reproducibility of science: unique identification of research resources in the biomedical literature. Peer J 1:e148. doi:10.7717/peerj.148

Vesterinen HV, Egan K, Deister A, Schlattmann P, Macleod MR, Dirnagl U (2011) Systematic survey of the design, statistical analysis, and reporting of studies published in the 2008 volume of the Journal of Cerebral Blood Flow and Metabolism. J Cereb Blood Flow Metab 31:1064-1072 